\title{
Spin-Orbital-Intertwined Nematic State in FeSe
}

\author{
J. Li, ${ }^{1}$ B. Lei, ${ }^{1}$ D. Zhao, ${ }^{1}$ L. P. Nie, ${ }^{1}$ D. W. Song, ${ }^{1}$ L. X. Zheng, ${ }^{1}$ S. J. Li ${ }^{1}$ B. L. Kang, ${ }^{1}$ \\ X. G. Luo, ${ }^{1,2,3,4,5}$ T. Wu®i ${ }^{1,2,3,4,5, *}$ and X. H. Chen ${ }^{1,2,3,4,5, \dagger}$ \\ ${ }^{1}$ Hefei National Laboratory for Physical Sciences at the Microscale and Department of Physics, \\ University of Science and Technology of China, Hefei, Anhui 230026, China \\ ${ }^{2}$ Key Laboratory of Strongly-coupled Quantum Matter Physics, Chinese Academy of Sciences, \\ University of Science and Technology of China, Hefei, Anhui 230026, China \\ ${ }^{3}$ CAS Center for Excellence in Superconducting Electronics (CENSE), Shanghai 200050, China \\ ${ }^{4}$ CAS Center for Excellence in Quantum Information and Quantum Physics, Hefei, Anhui 230026, China \\ ${ }^{5}$ Collaborative Innovation Center of Advanced Microstructures, Nanjing University, \\ Nanjing 210093, China
}

(Received 29 April 2019; revised manuscript received 13 December 2019; accepted 25 December 2019; published 13 February 2020)

\begin{abstract}
The importance of the spin-orbit coupling (SOC) effect in Fe-based superconductors (FeSCs) has recently been under hot debate. Considering the Hund's coupling-induced electronic correlation, the understanding of the role of SOC in FeSCs is not trivial and is still elusive. Here, through a comprehensive study of ${ }^{77} \mathrm{Se}$ and ${ }^{57} \mathrm{Fe}$ nuclear magnetic resonance, a nontrivial SOC effect is revealed in the nematic state of FeSe. First, the orbital-dependent spin susceptibility, determined by the anisotropy of the ${ }^{57} \mathrm{Fe}$ Knight shift, indicates a predominant role from the $3 d_{x y}$ orbital, which suggests the coexistence of local and itinerant spin degrees of freedom in the FeSe. Then, we reconfirm that the orbital reconstruction below the nematic transition temperature $\left(T_{\text {nem }} \sim 90 \mathrm{~K}\right.$ ) happens not only on the $3 d_{x z}$ and $3 d_{y z}$ orbitals but also on the $3 d_{x y}$ orbital, which is beyond a trivial ferro-orbital order picture. Moreover, our results also indicate the development of a coherent coupling between the local and itinerant spin degrees of freedom below $T_{\text {nem, }}$, which is ascribed to a Hund's coupling-induced electronic crossover on the $3 d_{x y}$ orbital. Finally, because of a nontrivial SOC effect, sizable in-plane anisotropy of the spin susceptibility emerges in the nematic state, suggesting a spin-orbital-intertwined nematicity rather than a simple spin- or orbital-driven nematicity. The present work not only reveals a nontrivial SOC effect in the nematic state but also sheds light on the mechanism of nematic transition in FeSe.
\end{abstract}

DOI: 10.1103/PhysRevX.10.011034

\section{INTRODUCTION}

Electronic correlation is widely accepted as a predominant physical origin for the production of the various quantum matter states in high-temperature superconductors (HTS), including cuprate superconductors [1] and Fe-based superconductors (FeSCs) [2-4]. In contrast, spin-orbit coupling (SOC) due to the relativistic motion of electrons has always been considered insignificant in these HTS families. However, a renaissance on the SOC in FeSCs is greatly promoted by the recent efforts to search for the

\footnotetext{
*wutao@ustc.edu.cn

†chenxh@ustc.edu.cn
}

Published by the American Physical Society under the terms of the Creative Commons Attribution 4.0 International license. Further distribution of this work must maintain attribution to the author(s) and the published article's title, journal citation, and DOI.
Subject Areas: Condensed Matter Physics, Strongly Correlated Materials, Superconductivity

influence of SOC on physical properties [4-22]. In general, SOC can intertwine spin and orbital degrees of freedom (d.o.f.) in solids and induce intriguing physical properties such as nontrivial topological band structure [23-26] and exotic superconducting pairing [27-29]. Recently, a nontrivial topological band structure has been successfully verified in iron-chalcogenide superconductors [19-21] that confirms the importance of SOC on the band structure in FeSCs. A natural follow-up question is how to understand the SOC effect on the various quantum matter states, such as nematic and superconducting states, due to electronic correlation in FeSCs [13-18].

FeSe is a good material for exploring the above issues. Because of its prominent electronic correlation, FeSe experiences a distinctive nematic transition compared with iron-pnictide superconductors [Fig. 1(a)] [30-32]. Below the nematic transition temperature $\left(T_{\text {nem }} \sim 90 \mathrm{~K}\right)$, only orbital order (without the presence of a long-range 
(a)

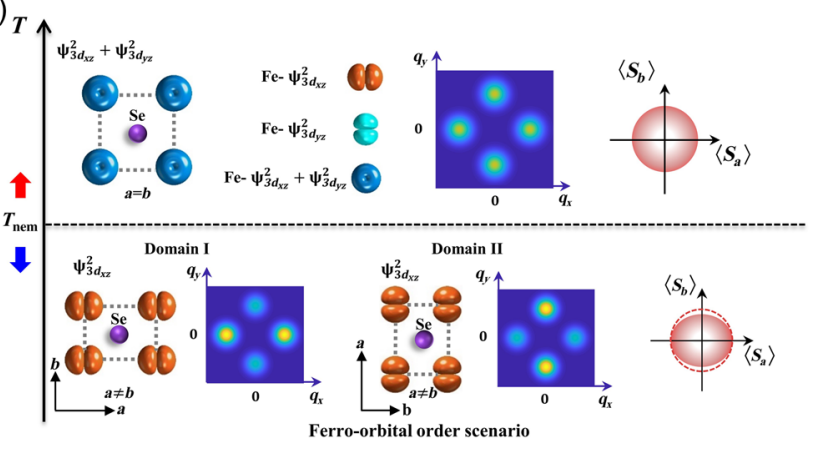

(b)

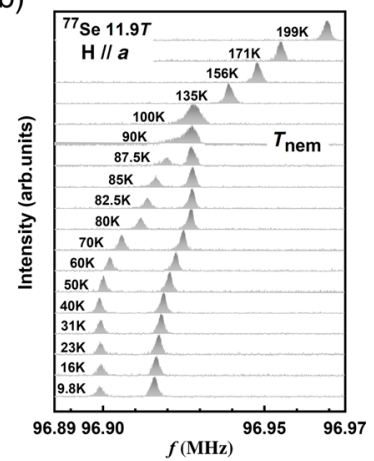

(c)

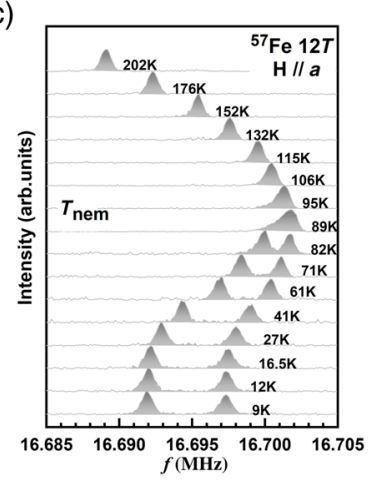

FIG. 1. NMR evidence for the Ising nematic transition in FeSe. (a) Illustrative diagram of the Ising nematic transition in FeSe. Above $T_{\text {nem }}$, the system is in a paramagnetic state with an isotropic dynamic spin correlation $\left[\left.\chi^{\prime \prime}(q)\right|_{(\pi, 0)}=\left.\chi^{\prime \prime}(q)\right|_{(0, \pi)}\right]$. Moreover, the $3 d_{x z}$ and $3 d_{y z}$ orbitals are also degenerate. In such a state, the localspin susceptibility is also isotropic within the $a b$ plane (indicated as a pink circle in the $\left\langle S_{a}\right\rangle$ vs $\left\langle S_{b}\right\rangle$ plot, in which $\left\langle S_{a}\right\rangle$ and $\left\langle S_{b}\right\rangle$ represent the local-spin polarization within the $a b$ plane). With the temperature lowering, a ferro-orbital ordering between the $3 d_{x z}$ and $3 d_{y z}$ orbitals accompanied by an orthorhombic lattice distortion $(a \neq b)$ takes place at $T_{\text {nem }}$ (indicated as the dashed black line). Two kinds of structural domains with different orbital polarizations appear equally, which always leads to a splitting in the NMR spectrum with $H \| a$ [Figs. 1(b) and 1(c)]. Because of SOC, the localspin susceptibility is expected to gain a slight anisotropy in each domain [36] (indicated as a pink ellipse in the $\left\langle S_{a}\right\rangle$ vs $\left\langle S_{b}\right\rangle$ plot). The dynamic spin correlation is coupled with the orbital order and shows an anisotropic nature. Here, whatever the driving force is for such an Ising nematic transition, the orbital order and anisotropic dynamic spin correlation are always twisted [37,38]. (b,c) Normalized NMR spectra for ${ }^{57} \mathrm{Fe}$ and ${ }^{77} \mathrm{Se}$ nuclei with $H \| a$. Below $T_{\text {nem }}$, a clear splitting of the line shape due to the formation of the nematic domains as discussed above is shown in the spectra for both the ${ }^{57} \mathrm{Fe}$ and ${ }^{77} \mathrm{Se}$ nuclei. It should be noted that the two splitting NMR lines for the ${ }^{77}$ Se nuclei show different intensities, which are caused by a partial detwinning due to unintentional strain from the tight NMR coils. We determine that such unintentional strain did not change any NMR results in the study and that the strain could be easily removed using loose NMR coils.

magnetic order) is observed down to the superconducting temperature $\left(T_{c} \sim 8.9 \mathrm{~K}\right)$ [33,34]. Currently, the underlying mechanism for such nematic transition is still under debate [35]. However, a sizable band splitting due to SOC

has been confirmed by recent angle-resolved photoemission spectroscopy (ARPES) measurement in FeSe. Moreover, such a SOC effect on band structure still survives in the presence of orbital order below $T_{\text {nem }}$ $[12,18]$. Furthermore, a significant anisotropy for spin excitations has also been observed in the nematic state with an inelastic neutron scattering experiment (INS) [13], suggesting a prominent role of SOC in the nematic state. All of the above facts indicate the important role of SOC in the physical properties of the nematic state in FeSe.

To further clarify the SOC effect and its implication in the nematic state, we conducted a site-selective nuclear magnetic resonance (NMR) study on a FeSe single crystal. In the previous NMR studies [33,34], only ${ }^{77}$ Se nuclei have been measurable, and the ${ }^{57} \mathrm{Fe}$ nuclei have been almost immeasurable due to their low abundance $(\sim 2.12 \%)$ and the small nuclear gyromagnetic ratio $\left({ }^{57} \gamma / 2 \pi=1.376 \mathrm{MHz} / \mathrm{T}\right)$. By synthesizing the high-quality ${ }^{57} \mathrm{Fe}$-enriched $(>98 \%) \mathrm{FeSe}$ single crystals, we successfully conducted NMR measurements on ${ }^{57} \mathrm{Fe}$ nuclei. The main findings from our present NMR results are summarized below. First, we reveal the predominant role of the $3 d_{x y}$ orbital on the orbital-dependent spin susceptibility, suggesting the importance of local spins for the local-spin susceptibility. Second, a significant involvement of the $3 d_{x y}$ orbital in orbital reconstruction below $T_{\text {nem }}$ is determined, which unambiguously excludes a trivial ferroorbital order in the nematic state. Third, our results also indicate a Hund's coupling-induced incoherent-to-coherent crossover on the $3 d_{x y}$ orbital below $T_{\text {nem. }}$. Finally, because of a nontrivial SOC effect, sizable in-plane anisotropy of the spin susceptibility is observed in the nematic state, which suggests a spin-orbital-intertwined nematicity in the FeSe.

\section{METHODS}

The ${ }^{57} \mathrm{Fe}$ isotope-enriched (>98\%) FeSe single crystalline samples were grown by a $\mathrm{KCl}-\mathrm{AlCl}_{3}$ flux method (see Supplemental Material [39]). The resistivity and the bulk magnetic susceptibility characterizations on the ${ }^{57} \mathrm{Fe}$ isotopeenriched samples are presented in the Supplemental Material [39]. These characterizations are the same as those of the normal samples with low abundance of the ${ }^{57} \mathrm{Fe}$ isotope. The details on the NMR measurement and relevant experimental setup are also described in the Supplemental Material [39]. Standard relaxation fitting functions and the fast Fourier transform (FFT) method were applied to analyze the NMR data. More details on the analysis of the NMR results are illustrated in the Supplemental Material [39].

\section{RESULTS}

\section{A. The Knight shift of the ${ }^{57} \mathrm{Fe}$ nuclei: Orbital-dependent spin susceptibility}

First, Figs. 1(b) and 1(c) show the frequency-swept NMR spectra of the ${ }^{57} \mathrm{Fe}$ and ${ }^{77} \mathrm{Se}$ nuclei for an in-plane 
magnetic field $(H \| a)$ in the temperature range of 8-200 K. A remarkable splitting of the NMR spectra is observed below $T_{\text {nem }}$ for both nuclei, which is ascribed to the formation of two orthogonal nematic domains with $H \| a$ and $H \| b[32-34,40,41]$. For the out-of-plane magnetic field, since both of the nematic domains are equivalent, there is no clear splitting for the NMR spectra with $H \| c$ below $T_{\text {nem }}$ (see Fig. S2 in Supplemental Material [39]). The absence of splitting in the ${ }^{57} \mathrm{Fe}$ NMR spectra with $H \| c$ excludes a translational symmetry breaking in one Fe unit cell due to exotic orbital ordering [42], supporting the identical orbital configuration at each Fe site (see Sec. S2 of the Supplemental Material [39]).

Then, we extract the Knight shift $K^{\alpha}(\alpha=a, b, c)$ from the peak position of the NMR spectra. The temperaturedependent Knight shift for both the ${ }^{77} \mathrm{Se}$ and ${ }^{57} \mathrm{Fe}$ nuclei are shown in Fig. 2. Generally, the Knight shift can be divided into two parts: $K^{\alpha}=K_{s}^{\alpha}+K_{\text {orb }}^{\alpha}(\alpha=a, b, c)$. Note that $K_{s}^{\alpha}$
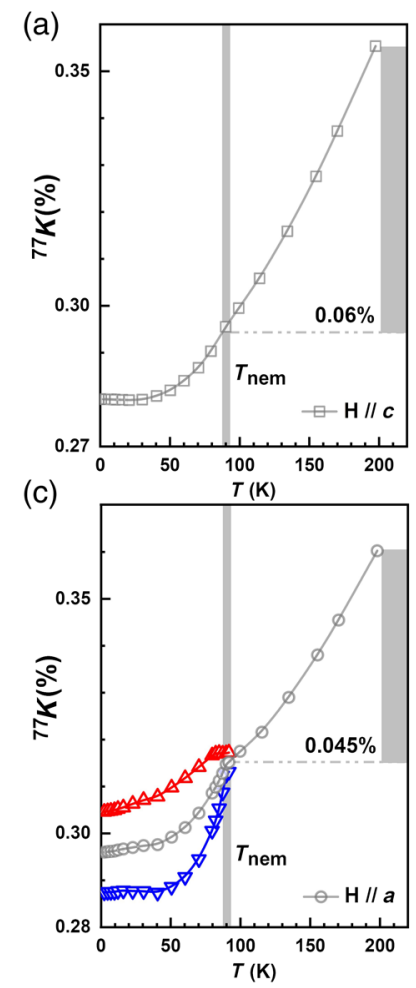

(d)
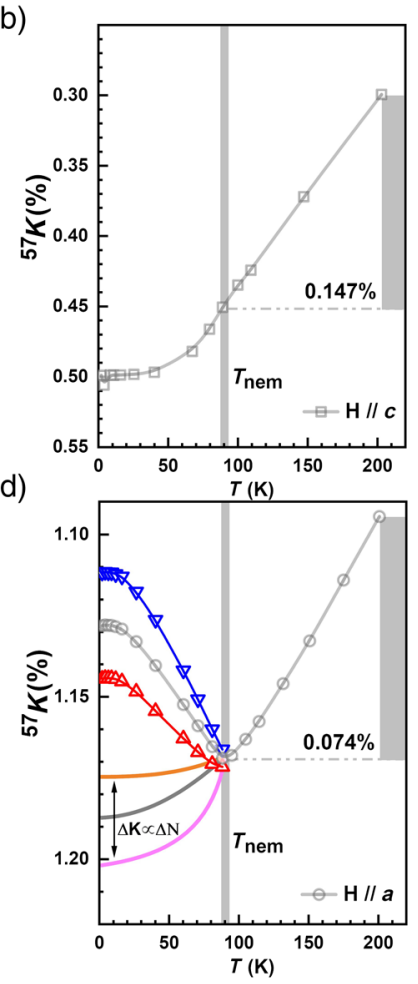

is the contribution from spin d.o.f. (spin shift), which is proportional to the local-spin susceptibility $\left(\chi_{s}\right)$, and it is usually temperature dependent. Here, $K_{\mathrm{orb}}^{\alpha}$ is the contribution from the orbital d.o.f. (orbital shift), which is usually temperature independent. By utilizing $K-\chi_{\text {bulk }}$ analysis, we can separate the spin shift and the orbital shift from the total Knight shift for both nuclei (see the Methods section of the Supplemental Material [39]). Above $T_{\text {nem }}$, although the bulk spin susceptibility is almost isotropic (see Fig. S3 in the Supplemental Material [39]), the spin shift for the ${ }^{57} \mathrm{Fe}$ nuclei exhibits a sizable anisotropy between the $a b$ plane and out-of-plane [Figs. 2(b) and 2(d)]. Since $K_{s}^{\alpha}=A^{\alpha} \chi_{s}^{\alpha}$, where $A^{\alpha}$ is the hyperfine coupling tensor between the nuclear and electronic spins, the anisotropic spin shift directly reflects the anisotropy of $A^{\alpha}$ in this case.

Next, we discuss the origin of the anisotropic $A^{\alpha}$ for the ${ }^{57} \mathrm{Fe}$ nuclei. Since the transferred hyperfine coupling is usually much less anisotropic than the on-site hyperfine

(e)

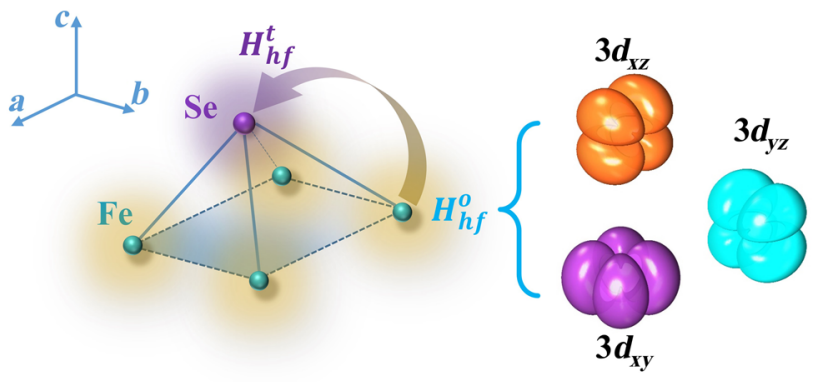

(f)

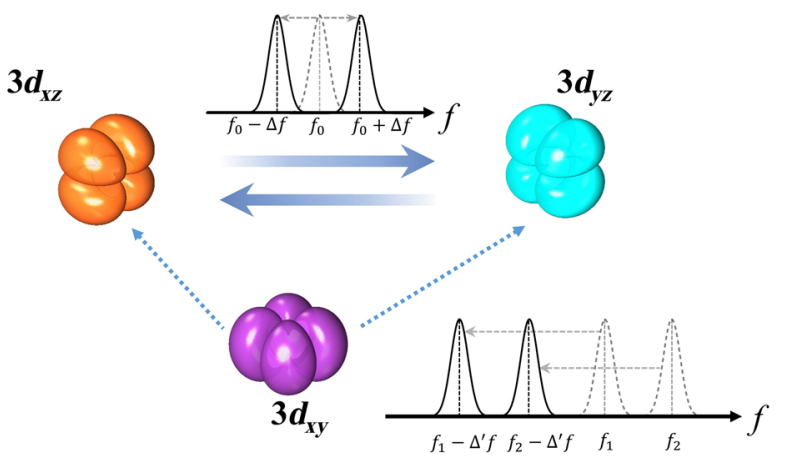

FIG. 2. Knight-shift evidence for orbital-dependent spin susceptibility and orbital order beyond a trivial ferro-orbital picture. (a)-(d) Temperature-dependent ${ }^{57} \mathrm{Fe}$ and ${ }^{77} \mathrm{Se}$ NMR Knight shift for $H \| a$ and $H \| c$. The red and blue open triangles represent the Knight shifts of the two splitting peaks below $T_{\text {nem. }}$. The open gray circles below $T_{\text {nem }}$ represent the averaged value of the splitting Knight shifts. The variation of the ${ }^{57} \mathrm{Fe}$ and ${ }^{77} \mathrm{Se}$ Knight shifts above $T_{\text {nem }}$ with $H \| a$ and $H \| c$ are indicated as filled gray rectangles. In panel (d), based on a two-orbital model with only $3 d_{x z}$ and $3 d_{y z}$ orbitals, the simulated temperature-dependent ${ }^{57} \mathrm{Fe}$ Knight shifts below $T_{\text {nem }}$ are also depicted (see the Methods section in the Supplemental Material for details [39]). The solid orange and magenta curves represent the two splitting Knight shifts. The gray bold line represents the average value of the two splitting Knight shifts. Here, $\Delta N$ is the electron population difference between the $3 d_{x z}$ and $3 d_{y z}$ orbitals due to the ferro-orbital order. (e) Sketches of the primary hyperfine interactions on ${ }^{57} \mathrm{Fe}$ and ${ }^{77} \mathrm{Se}$ nuclei. The hyperfine field on ${ }^{77} \mathrm{Se}$ is transferred from the Fe $3 d$ orbitals through the hybridization with the $4 p$ and $4 s$ orbitals at the Se sites $\left(H_{h f}^{t}\right)$. For the ${ }^{57} \mathrm{Fe}$ nuclei, the dominated hyperfine field is the on-site hyperfine coupling $\left(H_{h f}^{o}\right)$ (see main text). (f) The role of the $3 d_{x y}$ orbital on the Knight shift below $T_{\text {nem. }}$. An imbalance distribution of electrons on the $3 d_{x z}$ and $3 d_{y z}$ orbitals within a two-orbital model will only cause a splitting in the NMR spectra with $H \| a$. When considering the redistribution between the $3 d_{x y}$ and $3 d_{x z}\left(3 d_{y z}\right)$ orbitals, an overall shift to a lower resonant frequency is expected. 
coupling [Fig. 2(e)] [43], we then consider only the on-site hyperfine coupling as the origin of the anisotropic $A^{\alpha}$. Given a dominant dipolar hyperfine coupling, the anisotropic part of $A^{\alpha}$ strongly depends on the local $3 d$ orbital configuration at the ${ }^{57} \mathrm{Fe}$ sites (see the Methods section in the Supplemental Material [39]). In our case, if considering an orbital configuration with a dominant $3 d_{x z}$ or $3 d_{y z}$ character, we expect $A_{c}<A_{a}$. This result is inconsistent with our experimental result. Only a dominant $3 d_{x y}$ character can be fitted to the observed anisotropy for $A_{\alpha}$. This case indicates the predominant role of the $3 d_{x y}$ orbital for the local-spin susceptibility. From the theoretical perspective, for simplicity, the spin susceptibility can be understood by either an itinerant spin picture or a local-spin picture. In the itinerant spin picture, the spin susceptibility is due to Pauli paramagnetism and is proportional to the density of states (DOS) at the Fermi level. Our NMR results indicate that if such an itinerant spin picture is applicable in FeSe, the DOS at the Fermi level should mainly come from the $3 d_{x y}$ orbital. However, this case is in conflict with previous ARPES results, in which the primary energy band across the Fermi level has $3 d_{x z}$ or $3 d_{y z}$ characteristics [44-47]. The $3 d_{x y}$ orbital has much less spectral weight at the Fermi level. This result strongly suggests that the local spins need to be seriously considered together with the itinerant spins in this system. Although the Hund's coupling-induced orbital-selective electronic correlation can offer a possible mechanism for such coexistence of local and itinerant spins in FeSe [6,48-52], understanding the local spins is not straightforward. We will discuss this issue in more detail later.

In contrast to the ${ }^{57} \mathrm{Fe}$ nuclei, the spin shift for the ${ }^{77} \mathrm{Se}$ nuclei shows much less anisotropy. The hyperfine coupling tensor of the ${ }^{77} \mathrm{Se}$ is determined by the polarization of the $4 p$ and $4 s$ orbitals at the Se sites through the hybridization with the $3 d$ orbital at the $\mathrm{Fe}$ sites. Only the less-extended (hybridized) Se- $4 p$ orbital can provide an anisotropic hyperfine field on the ${ }^{77} \mathrm{Se}$. Thus, the weak anisotropy of spin shift at the ${ }^{77} \mathrm{Se}$ sites is expected, which makes the $3 d_{x y}$ orbital at the ${ }^{57} \mathrm{Fe}$ sites indistinguishable from the ${ }^{77} \mathrm{Se}$ NMR.

\section{B. Orbital reconstruction beyond a trivial ferro-orbital order}

With the above knowledge on the orbital-dependent local-spin susceptibility, we next discuss the results in the nematic state. As shown in Fig. 2(d), in addition to the well-known splitting of the NMR spectra due to the nematic order, the averaged in-plane Knight shift of the ${ }^{57} \mathrm{Fe}$ nuclei $\left[K^{a b}(T)\right]$ also exhibits a remarkable upturn behavior below $T_{\text {nem }}$, which is in sharp contrast to the Knight shift of the ${ }^{77} \mathrm{Se}$ nuclei. If we only consider a simple ferro-orbital order with a redistribution of the orbital population between $3 d_{x z}$ and $3 d_{y z}$ orbitals as suggested by previous ${ }^{77} \mathrm{Se}$ NMR results [33], the expected behavior of the averaged in-plane Knight shift for both ${ }^{57} \mathrm{Fe}$ and ${ }^{77} \mathrm{Se}$ nuclei should follow the same temperature dependence below $T_{\text {nem }}$ [see the caption in Fig. 2(d)]. This case suggests that a simple ferro-orbital order involving only $3 d_{x z}$ and $3 d_{y z}$ orbitals is insufficient to understand the present ${ }^{57} \mathrm{Fe}$ NMR results. Considering previous ARPES results $[44,46,53]$, it strongly supports a necessary change of the $3 d_{x y}$ orbital in the nematic state. For simplicity, we consider one possible change of the population on the $3 d_{x y}$ orbital as shown in Fig. 2(f). In this case, the upturn behavior of the averaged in-plane Knight shift of the ${ }^{57} \mathrm{Fe}$ nuclei is due to the population reduction on the $3 d_{x y}$ orbital in the nematic state. However, such an explanation only makes sense within an itinerant spin picture in consideration of the Fermi surface reconstruction, which is still too simple to elucidate the underlying physics for the ${ }^{57} \mathrm{Fe}$ Knight shift below $T_{\text {nem. }}$.

What are the underlying physics that accounted for such a change of the $3 d_{x y}$ orbital? As we mentioned before, the orbital-dependent spin susceptibility determined by the ${ }^{57} \mathrm{Fe}$ Knight shift is beyond a simply itinerant spin picture, and the local spins need to be considered as a predominant origin. If considering the Hund's coupling-induced electronic correlation in FeSCs, such coexistence of the itinerant and local spins is a natural consequence of the so-called orbital-selective electronic correlation $[48,49,54]$. In FeSCs, the $3 d_{x y}$ orbital always presents a stronger electronic correlation than the others, and it should play a key role for the local spins [52], which is also supported by the present ${ }^{57} \mathrm{Fe}$ NMR results. However, considering the hybridization among various orbitals, a universal incoherent-to-coherent electronic crossover is also a manifestation of the Hund's coupling-induced electronic correlation [48], which has already been verified in FeSCs [49-51]. Such electronic crossover has also been observed in most iron-chalcogenide superconductors by ARPES [55]. One main feature for such electronic crossover in these ironchalcogenide superconductors is that, with the emergence of the $3 d_{x y}$ band with decreasing temperature, a hybridization gap due to the cross of $3 d_{x y}$ and $3 d_{x z}$ bands opens around the $\mathrm{M}$ point. We propose that the change of the $3 d_{x y}$ orbital suggested by the present ${ }^{57} \mathrm{Fe}$ Knight shift could be ascribed to a similar incoherent-to-coherent electronic crossover on the $3 d_{x y}$ orbital. Very recently, two ARPES experiments indicated that, since the nematic band shifting enables a band crossing between $3 d_{x y}$ and $3 d_{x z}$ bands at the M point, a hybridization gap occurs below $T_{\text {nem }}[56,57]$, which may further promote the incoherent-to-coherent crossover of the $3 d_{x y}$ band. Therefore, our NMR result suggests a close correlation between the nematic transition and incoherent-to-coherent crossover of the $3 d_{x y}$ band. Further theoretical calculation is needed to verify the above picture with a quantitative explanation of the results of the ${ }^{57} \mathrm{Fe}$ Knight shift. 


\section{Evidence for spin-space anisotropy: Uniform spin susceptibility}

Next, we turn to the detailed analysis of the splitting of NMR spectrum under $H \| a$ below $T_{\text {nem. }}$. As we discussed before, the splitting of the NMR spectrum is due to the formation of two orthogonal nematic domains with $H \| a$ and $H \| b$. Hence, measuring the splitting of the NMR spectrum is actually equal to measuring the in-plane anisotropy of the Knight shift, which is defined as $\Delta K(T)=K_{s}^{a}(T)-K_{s}^{b}(T)$. Since $K_{s}^{\alpha}=A_{s}^{\alpha} \chi_{s}^{\alpha}$, both of the in-plane anisotropies of the hyperfine coupling constant $\left[\Delta A_{s}(T)={ }^{57,77} A_{s}^{a}(T)-{ }^{57,77} A_{s}^{b}(T)\right]$ and the localspin susceptibility $\left[\Delta \chi_{s}(T)=\chi_{s}^{a}(T)-\chi_{s}^{b}(T)\right]$ contribute to $\Delta K(T)$. When $\Delta K(T) /\left(K^{a b}(T)\right) \ll 1$ and when $K^{a b}(T)$ is weakly temperature dependent below $T_{\text {nem }}, \Delta K(T)$ can be approximately expressed as a linear combination of $\Delta A_{s}(T)$ and $\Delta \chi_{s}(T)$. A previous ${ }^{77} \mathrm{Se}$ NMR study determined that $\Delta^{77} K(T)$ is linearly related to the order parameter of the orbital ordering transition $\left[\Delta_{o}(T)\right]$ [33]. This conclusion is correct only when both $\Delta^{77} A_{s}(T)$ and $\Delta \chi_{s}(T)$ are proportional to $\Delta_{o}(T)$. Considering an orbital-driven (only the orbital d.o.f. is activated) ferro-orbital order, a finite SOC will only produce a negligible $\Delta \chi_{s}(T)$, which should be proportional to $\Delta_{o}(T)$. This case was verified by the RPA calculation in $\mathrm{BaFe}_{2} \mathrm{As}_{2}$ by only considering a ferroorbital order without invoking spin d.o.f. [36]. In this situation, the above conjecture is naturally satisfied. It has been used as a proof to support the orbital-driven nematic order in FeSe [33]. However, the present result proves that $\Delta \chi_{s}(T)$ has a sizable value and that it is not linearly coupled to $\Delta_{o}(T)$.

As shown in Fig. 3(a), when we plot the $\Delta K(T)$ for both the ${ }^{77} \mathrm{Se}$ and ${ }^{57} \mathrm{Fe}$ nuclei together, there is a clear difference between $\Delta^{77} K(T)$ and $\Delta^{57} K(T)$ below a characteristic temperature $T^{*} \sim 75 \mathrm{~K}$. Especially for $\Delta^{77} K(T)$, it starts to decrease below about $60 \mathrm{~K}$. This behavior has already been observed in a previous NMR measurement, but its origin is still elusive $[33,34]$. A strong temperature-dependent in-plane spin susceptibility $\left[\overline{\chi_{s}^{a b}}(T)\right]$ can give a simple explanation [58]. However, such an explanation seems unlikely because both the bulk spin susceptibility and the spin shift are almost temperature independent below $60 \mathrm{~K}$ (see Figs, S3 and S4 in the Supplemental Material [39]). Furthermore, we also plot the temperature-dependent orthorhombic distortion of the lattice $\delta(T)$ in Fig. 3(a), which is proportional to $\Delta_{o}(T)$ [59]. Neither $\Delta^{77} K(T)$ nor $\Delta^{57} K(T)$ could be scaled to $\delta(T)$. The key to solving the discrepancy between $\Delta^{77} K(T)$ and $\Delta^{57} K(T)$ is to consider a nontrivial $\Delta \chi_{s}(T)$ that is not simply proportional to $\Delta_{o}(T)$. As we discussed before, $\Delta^{57,77} K(T)$ can be expressed as a linear combination of both $\Delta A_{s}(T)$ and $\Delta \chi_{s}(T)$ as shown in [33]:

$$
\begin{aligned}
\Delta^{57,77} K(T)= & { }^{57,77} A_{s}^{a}(T) \chi_{s}^{a}(T)-{ }^{57,77} A_{s}^{b}(T) \chi_{s}^{b}(T) \\
= & 1 / 2\left\{\left[{ }^{57,77} A_{s}^{a}(T)-{ }^{57,77} A_{s}^{b}(T)\right]\left[\chi_{s}^{a}(T)+\chi_{s}^{b}(T)\right]\right. \\
& \left.+\left[{ }^{57,77} A_{s}^{a}(T)+{ }^{57,77} A_{s}^{b}(T)\right]\left[\chi_{s}^{a}(T)-\chi_{s}^{b}(T)\right]\right\} \\
= & 1 / 2\left\{\overline{\chi_{s}^{a b}}(T) \Delta_{o}(T)+\overline{{ }^{57,77} A_{s}^{a b}}(T) \Delta \chi_{s}(T)\right\} .
\end{aligned}
$$

Because both $\overline{\chi_{s}^{a b}}(T)$ and $\overline{{ }^{57,77} A_{s}^{a b}}(T)$ are weakly temperature dependent below $T_{\text {nem }}$, the expression can be simplified as $\Delta^{57,77} K(T) \simeq 1 / 2\left\{\overline{\chi_{s}^{a b}} \Delta_{o}(T)+\overline{{ }^{57,77} A_{s}^{a b}} \Delta_{\chi_{s}}(T)\right\}$. Since $A_{s}^{\alpha}(T)$ can be determined by the $K_{s}^{\alpha}(T)-\chi_{\text {bulk }}(T)$ plot, we find that the sign of the second term in the expression is different for ${ }^{77} \mathrm{Se}$ and ${ }^{57} \mathrm{Fe}$ (see Figs. S4 and S5 in the Supplemental Material [39]). When $\Delta \chi_{s}(T)$ is not simply proportional to $\Delta_{o}(T), \Delta K(T)$ for both of the nuclei will naturally show different temperature dependences, including the decrease in $\Delta^{77} K(T)$. Based on the above analysis, we can separate $\Delta_{o}(T)$ [Fig. 3(b)] and $\Delta \chi_{s}(T)$ [Fig. 3(c)] from $\Delta^{57,77} K(T)$ (see Supplemental Material [39]). The extracted $\Delta_{o}(T)$ is proportional to $\delta(T)$ as expected. In contrast, the extracted $\Delta \chi_{s}(T)$ is almost negligible just below $T_{\text {nem. }}$. It starts to linearly increase only below $T^{*} \sim 75 \mathrm{~K}$ and level off to a sizable value below about $20 \mathrm{~K}$. A rough estimation for $\Delta \chi_{s}(T) / \chi_{s}(T)$ is about $14 \%$ at around $10 \mathrm{~K}$ (see Sec. S6 in the Supplemental Material [39]). Such sizable anisotropy is not consistent with a simply orbital-driven nematic order $[33,36]$, suggesting a novel spin-orbital intertwined nematic state. This result is another main finding of the present work.

Recently, the bulk magnetic susceptibility measurements on a FeSe single crystal with a uniaxial strain have also revealed similar in-plane anisotropy of the spin susceptibility [60]. However, the starting temperature for such an in-plane anisotropy was observed slightly above $T_{\text {nem }}$. Since our NMR experiment is performed without any external strain, the external strain effect might be a cause for such discrepancy in the starting temperature. However, the in-plane anisotropy in bulk magnetic susceptibility was ascribed to a short-range stripe magnetic order [60]. In this case, the short-range magnetic order will strongly broaden the linewidth of the NMR spectrum, which cannot give a clear line splitting. This result is in conflict with the present NMR experiment, in which the anisotropy of the local-spin susceptibility only affects the uniform spin shift rather than the linewidth (see Sec. S7 in Supplemental Material [39]). Therefore, the possibility of a short-range magnetic order can be ruled out.

\section{Evidence for spin-space anisotropy: Dynamic spin susceptibility}

In order to further verify the spin-space anisotropy in the nematic state, we also study the nuclear spin-lattice relaxation. In general, the quantity of $1 / T_{1} T$ (spin-lattice relaxation rate divided by $T$ ) measures the strength of 

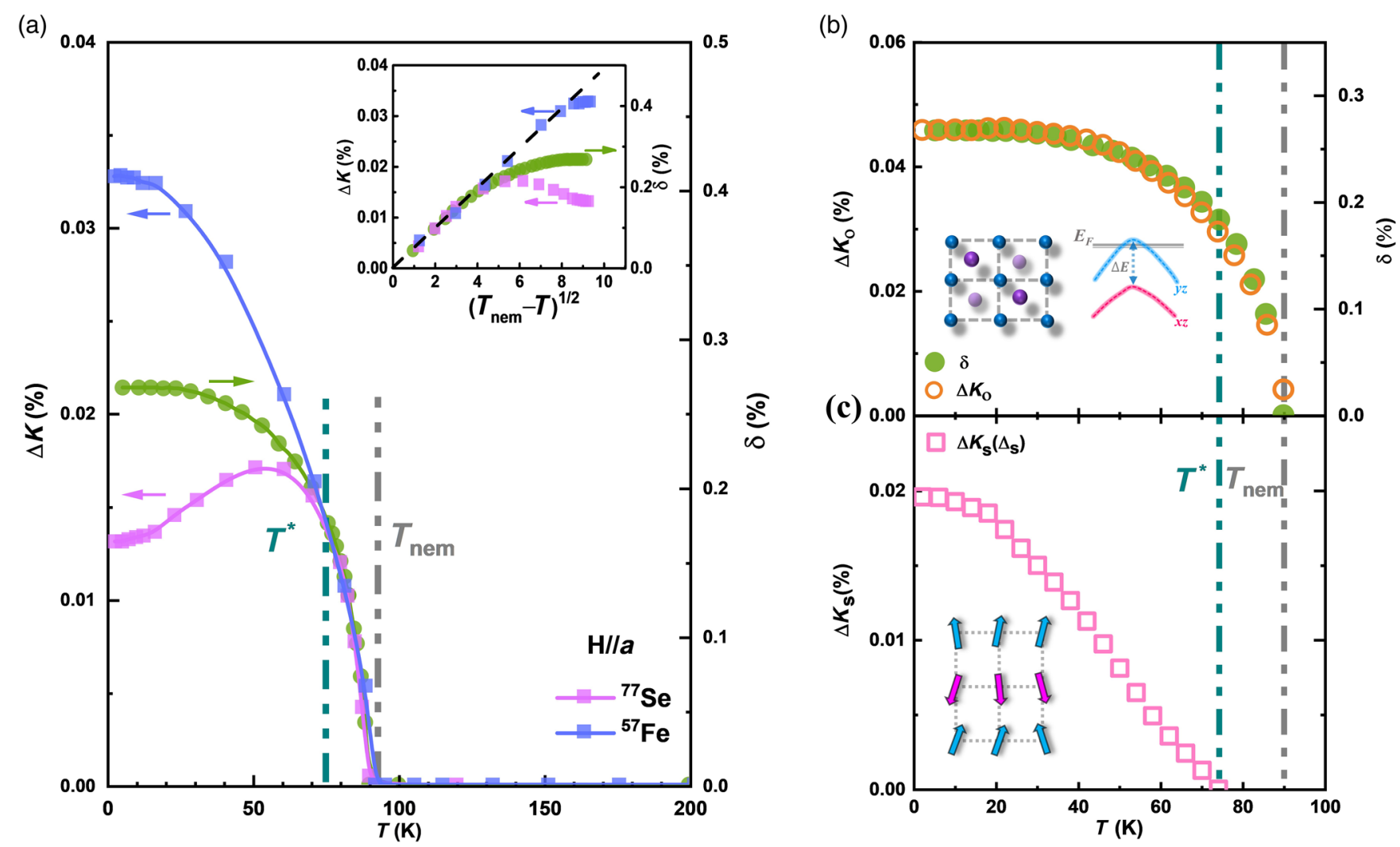

FIG. 3. Knight-shift evidence for a sizable anisotropy in spin space: Temperature-dependent splitting of the Knight shift. (a) Temperature evolution of the in-plane Knight-shift anisotropy of ${ }^{57} \mathrm{Fe}$ and ${ }^{77} \mathrm{Se}$. The experimental data are shown as solid light-blue squares $\left({ }^{57} \mathrm{Fe}\right)$ and solid light-magenta squares $\left({ }^{77} \mathrm{Se}\right)$. The $T$-dependent lattice orthorhombicity $\delta(T)(\delta=(a-b) /(a+b))$ measured through synchrotron high-energy x-ray diffraction in Ref. [42] is also reproduced with solid dark-green circles. The inset (top right) shows the proportional relationship in the proximity of the second-order phase transition: $\left(\Delta^{57} K, \Delta^{77} K, \delta(T) \propto\left(T_{\text {nem }}-T\right)^{1 / 2}\right)$. The discrepancy below $T^{*}$ is also clearly shown. Below about $20 \mathrm{~K}$, all three quantities are saturated. The solid lines are guides for the eye. (b) The temperature-dependent $\Delta K_{o}(T)$ and lattice orthorhombicity $\delta(T)$. The $\Delta K_{o}(T)$ and $\delta(T)$ are marked as open orange circles and filled green circles. [The data are interpolated from panel (a)]. The inset shows the cartoon picture for the orbital-related band splitting and orthorhombic structural distortion. (c) The temperature-dependent $\Delta K_{s}(T)$ (or anisotropy of the local-spin susceptibility). Interestingly, the $\Delta K_{s}(T)$ starts to arise only below $T^{*}$ instead of immediately below $T_{\text {nem. }}$. The inset shows a cartoon depiction of the anisotropic spin polarization in the Ising nematic state. The extracted $\Delta K_{s}(T)$ is interpolated from the original data shown in panel (a) with spline interpolation methods.

low-energy spin fluctuations with $1 / T_{1} T \sim \Sigma_{q} \gamma_{n}^{2}\left|A_{\perp}(q)\right|^{2}$ $\left[\chi^{\prime \prime}(q, \omega) / \omega\right]$, where $\chi^{\prime \prime}(q, \omega)$ and $A_{\perp}(q)$ are the $q$-dependent dynamic spin susceptibility and the transverse hyperfine form factor, respectively [41]. When the $\chi^{\prime \prime}(q, \omega)$ exhibits a similar spin-space anisotropy as the uniform spin susceptibility, the $1 / T_{1} T$ is a good indicator. In addition to the spin-space anisotropy, the anisotropic spin excitations in $k$ space due to stripe-type spin fluctuations [Fig. 1(a)] [38], which are always twisted with orbital order in the nematic state, could also affect the anisotropy of $1 / T_{1} T$ (see Sec. S8 in the Supplemental Material [39]). This result has already been verified in $\mathrm{NaFe}_{1-\mathrm{x}} \mathrm{Co}_{\mathrm{x}}$ As [41]. In this case, a specific $A_{\perp}(q)$ is necessary to transfer the $k$-space anisotropy into the anisotropy of $1 / T_{1} T$. Otherwise, the anisotropic spin excitation in $k$ space cannot have a significant effect on the anisotropy of $1 / T_{1} T$. After careful analysis on the $A_{\perp}(q)$ for both the ${ }^{77} \mathrm{Se}$ and ${ }^{57} \mathrm{Fe}$ sites, we find that only the ${ }^{77} \mathrm{Se}$ nuclei can satisfy the above requirement for $A_{\perp}(q)$. Therefore, the anisotropy of $1 / T_{1} T$ for ${ }^{77} \mathrm{Se}$ nuclei can be affected by the anisotropy in both spin and $k$ space, but the anisotropy of $1 / T_{1} T$ for ${ }^{57} \mathrm{Fe}$ nuclei can be affected only by the spin-space anisotropy.

As shown in Fig. 4(a), the temperature-dependent $1 / T_{1} T$ for each splitting NMR line shows a similar behavior but with different values below $T_{\text {nem }}$. This result means that the $1 / T_{1} T$ for each structural domain is anisotropic within the $a b$ plane. If we define $\Delta 1 / T_{1} T=\left[\left(1 /\left.T_{1} T\right|_{H \| a}-\right.\right.$ $\left.\left.1 /\left.T_{1} T\right|_{H \| b}\right) /\left(1 /\left.T_{1} T\right|_{H \| a}+1 /\left.T_{1} T\right|_{H \| b}\right)\right]$, it is clear that the temperature-dependent $\Delta 1 / T_{1} T$ is distinct for the ${ }^{77} \mathrm{Se}$ and ${ }^{57} \mathrm{Fe}$ nuclei [Fig. 4(b)] as we expected. For the ${ }^{57} \mathrm{Fe}$ nuclei, the temperature-dependent $\Delta 1 / T_{1} T$ only becomes significant below $T^{*}$ and roughly follows the same temperature dependence as that for $\Delta K_{s}(T)$, which supports a spinspace anisotropy from the dynamic spin susceptibility. 
(a)

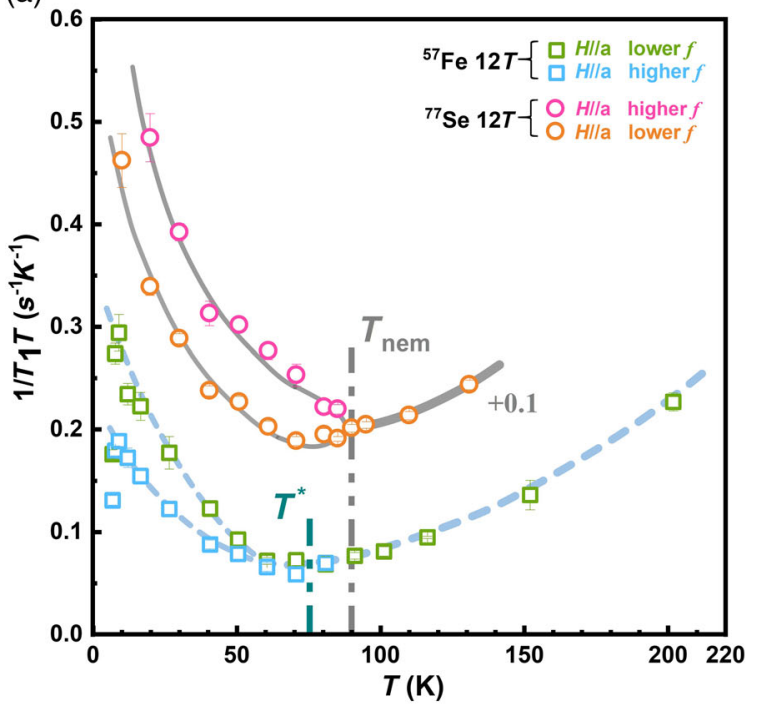

(b)

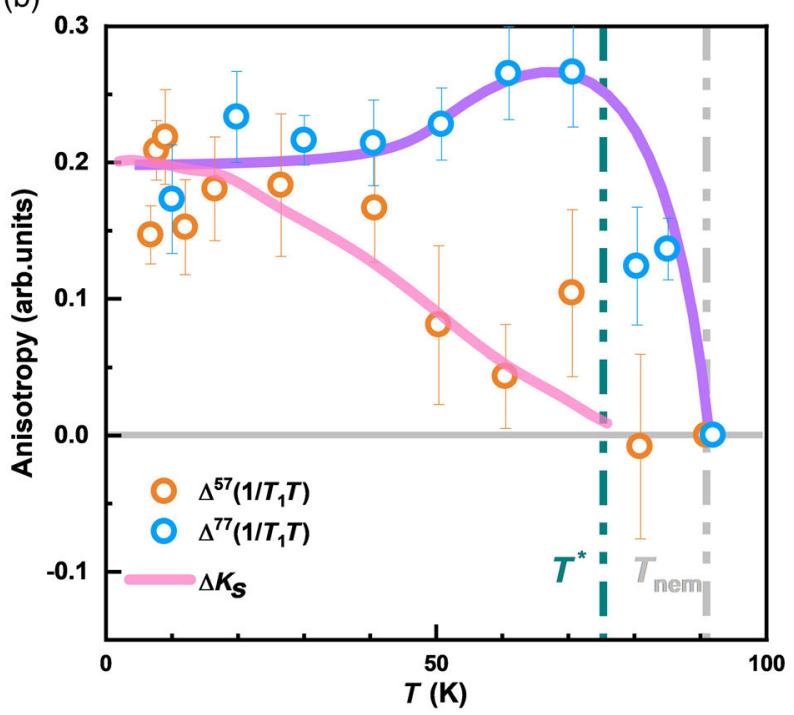

FIG. 4. Anisotropic nuclear spin-lattice relaxation in the nematic state: Temperature-dependent anisotropy in the spin-lattice relaxation rate divided by $T$. (a) Nuclear spin-lattice relaxation rate divided by $T$ versus the temperature on the ${ }^{57} \mathrm{Fe}$ and ${ }^{77} \mathrm{Se}$ sites with $H \| a$. Note that $1 / T_{1} T$ of ${ }^{77} \mathrm{Se}$ in different domains are marked as open magenta and orange circles. The $1 / T_{1} T$ of ${ }^{57} \mathrm{Fe}$ in different domains are marked as open green and blue squares. In order to compare with ${ }^{57} \mathrm{Fe}$ and ${ }^{77} \mathrm{Se}$, the magnitudes of the $1 / T_{1} T$ for ${ }^{77} \mathrm{Se}$ are shifted up by 0.1 . Interestingly, the splitting of $1 / T_{1} T$ for ${ }^{57} \mathrm{Fe}$ and ${ }^{77} \mathrm{Se}$ starts from different temperatures. (b) The temperature-dependent anisotropy in the $1 / T_{1} T$ is defined as $\Delta 1 / T_{1} T=\left[\left(1 /\left.T_{1} T\right|_{H \| a}-1 /\left.T_{1} T\right|_{H \| b}\right) /\left(1 /\left.T_{1} T\right|_{H \| a}+1 /\left.T_{1} T\right|_{H \| b}\right)\right]$. The anisotropies for both ${ }^{57} \mathrm{Fe}$ and ${ }^{77} \mathrm{Se}$

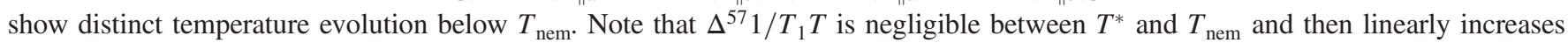
below $T^{*}$, which is scaled with $\Delta_{s}(T)$. In stark contrast to $\Delta^{57} 1 / T_{1} T, \Delta^{77} 1 / T_{1} T$ shows a rapid increase immediately below $T_{\text {nem }}$ and then follows a broad peak around $65 \mathrm{~K}$. Finally, $\Delta^{77} 1 / T_{1} T$ becomes saturated with temperature below $50 \mathrm{~K}$. As discussed in the main text, such a difference between ${ }^{57} \mathrm{Fe}$ and ${ }^{77} \mathrm{Se}$ is related to the detailed information on both $A_{h f}(q)$ and $\chi^{\prime \prime}(q, \omega)$ (see Sec. S8 in the Supplemental Material [39]).

In contrast, for the ${ }^{77} \mathrm{Se}$ nuclei, the temperature-dependent $\Delta 1 / T_{1} T$ shows a nonmonotonic temperature dependence because the effect of the $k$-space and spin-space anisotropies on $\Delta 1 / T_{1} T$ is not simply additive. Just below $T_{\text {nem }}$, the $\Delta 1 / T_{1} T$ is dominated by the anisotropic spin excitations in $k$ space, which is always twisted with the orbital order and which shows a rapid increase similar to that in $\Delta K_{o}(T)$. When the temperature is lower than $T^{*}$, the contribution from the spin-space anisotropy comes into play. This case leads to a slight decrease in $\Delta 1 / T_{1} T$ for the ${ }^{77}$ Se nuclei (see Sec. S8a in the Supplemental Material [39]). In summary, the above results for the nuclear spin-lattice relaxation also confirm a significant spin-space anisotropy in the dynamic spin susceptibility, which further supports a nontrivial SOC effect in the nematic state of the FeSe.

\section{DISCUSSION AND CONCLUSIONS}

Although the SOC always leads to a twist of spin and orbital d.o.f., the sizable spin-space anisotropy observed in the present study indicates a nontrivial SOC effect in the nematic state. In FeSCs, the driving force for a nematic transition is still under debate [35]. Because of the absence of a magnetic order, a simple orbital-driven nematic order has been proposed to understand the nematic transition in FeSe [33]. In this case, a trivial SOC effect is expected with a negligible in-plane anisotropy for spin susceptibility, which should be simply scaled with the orbital order $[36,60]$. Clearly, this result is inconsistent with our observation. However, if we consider a simple spin-driven nematic order, it would also be difficult to understand the distinct temperature dependence of the anisotropy of spin susceptibility from the nematic order. All these facts indicate that a simple spin or orbital-driven scenario is not enough to account for these new NMR results.

In this section, we discuss the possible role of Hund's coupling in such a nontrivial SOC effect. A distinguished character for the Hund's coupling-induced electronic correlation is orbital selectivity $[48,49,52,54]$. As discussed before, the orbital-dependent spin susceptibility determined by the ${ }^{57} \mathrm{Fe}$ NMR result confirms such orbital-selective electronic correlation and suggests a predominant role of localized spin for the local-spin susceptibility. For this situation, a simplified picture for understanding the origin of spin susceptibility is based on the coexistence of both local and itinerant spins. Although such a picture is quite practical for explaining the results of the ${ }^{57} \mathrm{Fe}$ Knight shift, it also highlights another fundamental issue for FeSCs, which is how to understand the origin of localized spins in FeSCs. Answering this question is beyond the scope of the present work. Hence, we only introduce a promising 
approach that is based on an empirical two-fluid model for FeSCs [52,61-63] to discuss this issue. In this model, the total electronic spectral weight is separated into two distinct parts. One part is relatively coherent, and it is related to the itinerant spins. The other part is relatively incoherent, and it is related to the local spins. The inter-orbital Hund's coupling leads to an on-site ferromagnetic coupling between these itinerant spins and the localized spins. Based on the above empirical model, we propose a simple picture to understand the above nontrivial SOC effect in the nematic state. As shown in Fig. 5, as the incoherent-tocoherent crossover on the $3 d_{x y}$ orbital is developing below $T_{\text {nem }}$, a coherent on-site ferromagnetic coupling between localized spins and itinerant spins is also simultaneously built up. This case means that the electronic system evolves from an incoherent state with two spin fluids into a coherent state with a single spin fluid. In such a coherent state, although the orthorhombicity due to orbital order is less than $0.3 \%$, the SOC induces a strong coupling between the spin and orbital d.o.f., which gives rise to sizable in-plane anisotropy of the local-spin susceptibility. This result is ascribed to a nontrivial SOC effect due to the Hund's coupling-induced electronic correlation, which is also the manifestation of a spin-orbital-intertwined nematic state.

Following the above scenario, the superconducting state may also be strongly affected by SOC. Recently, an extremely anisotropic superconducting gap has been observed in bulk FeSe [16,17]. Moreover, the superconducting pairing has also been revealed as orbital selective [64]. All of these experimental results may have a strong link with the spin-orbital-intertwined nematicity observed in the present work. Interestingly, a previous NMR study found that the Knight shift stayed almost constant across the superconducting transition temperature $[33,34]$, which leads to an argument for $p$-wave pairing [17]. This case is worthy of further exploration by considering a nontrivial

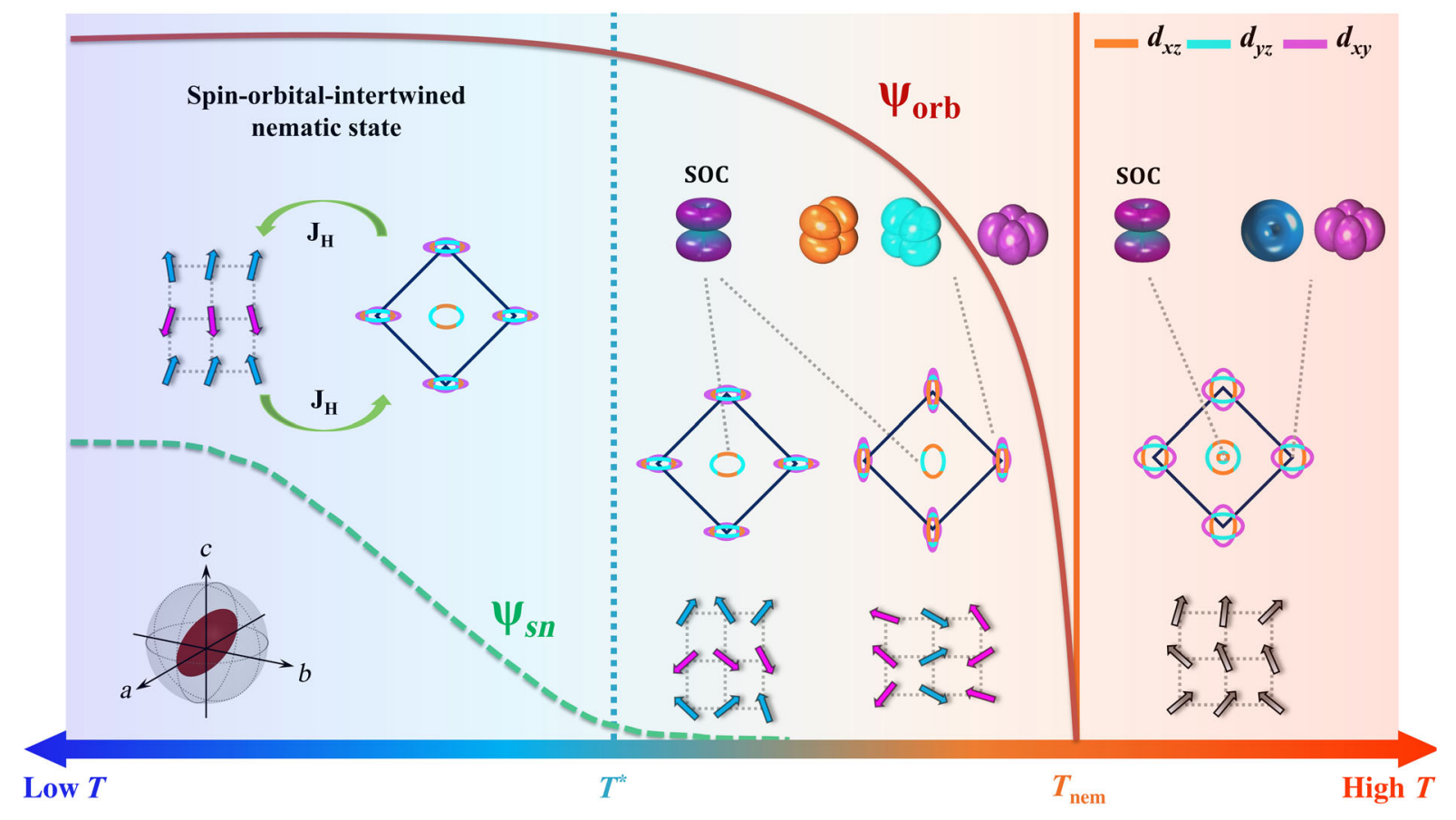

FIG. 5. A proposed physical scenario for the spin-orbital intertwined nematic state in FeSe. We adopt an empirical two-fluid model (a detailed description is presented in the main text) to explain our proposed scenario. The coherent part in this model contributes to the Fermi surface, which is related to itinerant spin d.o.f. The incoherent part in this model stands for the local-spin d.o.f. with a predominant $3 d_{x y}$ character. These two parts are coupled by Hund's coupling. Above $T_{\text {nem }}$, the coupling between the local and itinerant spins is incoherent. A negligible SOC effect was verified by the recent INS experiments [13]. A nearly isotropic temperature-dependent bulk magnetic susceptibility is also confirmed, which is consistent with a negligible SOC effect (see Supplemental Material [39]). Below $T_{\text {nem }}$, an orbital order driven by nematic transition emerges, and the corresponding temperature-dependent order parameter $\left(\Psi_{\text {orb }}\right)$ obeys a standard second-order phase transition. Such orbital order breaks the rotational symmetry of the lattice and leads to a simultaneous Fermi surface reconstruction. Considering a finite SOC, the spin d.o.f. should also break the rotational symmetry due to orbital order. In this case, if the spin susceptibility is dominated by the local spins, only a negligible in-plane anisotropy $\left(\Psi_{s n}\right)$ can be observed through local-spin susceptibility. However, a sizable in-plane anisotropy of spin susceptibility is verified by the NMR experiment in this research (ellipsoid with a long axis along the $a$ and $c$ crystallographic directions at the bottom-left inset), which is ascribed to an emergent coherent coupling between local and itinerant spins by Hund's coupling below $T_{\text {nem. }}$. A remarkable change of $\Psi_{s n}$ only happens below $T^{*}$, which also proves that such sizable in-plane anisotropy is related not only to the orbital order but also to the Hund's couplinginduced coherence. For more details, see the main text. 
SOC effect. In addition, another possible SOC effect on the superconducting state has also been suggested by a recent high-field study [65]. Currently, the SOC effect on the superconducting state is still elusive, which calls for further studies.

In conclusion, by utilizing a site-selective NMR measurement, the Hund's coupling-induced orbital-selective electronic correlation is reconfirmed by investigating the orbital-dependent local-spin susceptibility in FeSe, suggesting a predominant role for the $3 d_{x y}$ orbital in spin susceptibility. This result also indicates that the itinerant spins alone were not enough to account for spin susceptibility and that local spins should be seriously considered as a predominant origin. Furthermore, the temperature evolution of the orbital-dependent local-spin susceptibility reveals a remarkable change of the $3 d_{x y}$ orbital in the nematic state, which is definitely beyond a trivial ferroorbital order picture. This result also enables us to propose a Hund's coupling-induced incoherent-to-coherent crossover on the $3 d_{x y}$ orbital, which may have a close correlation with a recently discovered hybridization gap around the $\mathrm{M}$ point. Finally, because of a nontrivial SOC effect, sizable in-plane anisotropy of the spin susceptibility is observed in the nematic state, which suggests a spin-orbital-intertwined nematicity rather than a simple spin- or orbital-driven nematicity in the FeSe.

\section{ACKNOWLEDGMENTS}

We thank Y. Li, F. Wang, R. Yu, G. Chen, K. Jin, X. L. Dong, R. Zhou, M.-H. Julien, R. M. Fernandes, J. Schmalian, and Z. Y. Weng for stimulating discussions. This work was supported by the National Natural Science Foundation of China (Grants No. 11888101 and No. 11522434), the National Key R\&D Program of the MOST of China (Grants No. 2016YFA0300201 and No. 2017YFA0303000), the Strategic Priority Research Program of the Chinese Academy of Sciences (Grant No. XDB25000000), and the Anhui Initiative in Quantum Information Technologies (Grant No. AHY160000).

[1] P. A. Lee, N. Nagaosa, and X.-G. Wen, Doping a Mott Insulator: Physics of High-Temperature Superconductivity, Rev. Mod. Phys. 78, 17 (2006).

[2] P. J. Hirschfeld, M. M. Korshunov, and I. I. Mazin, Gap Symmetry and Structure of Fe-Based Superconductors, Rep. Prog. Phys. 74, 124508 (2011).

[3] Q. Si, R. Yu, and E. Abrahams, High-Temperature Superconductivity in Iron Pnictides and Chalcogenides, Nat. Rev. Mater. 1, 16017 (2016).

[4] R. M. Fernandes and A. V. Chubukov, Low-Energy Microscopic Models for Iron-Based Superconductors: A Review, Rep. Prog. Phys. 80, 014503 (2017).
[5] J. Wu, P. Phillips, and A. H. C. Neto, Theory of the Magnetic Moment in Iron Pnictides, Phys. Rev. Lett. 101, 126401 (2008).

[6] M. H. Christensen, J. Kang, B. M. Andersen, I. Eremin, and R. M. Fernandes, Spin Reorientation Driven by the Interplay between Spin-Orbit Coupling and Hund's Rule Coupling in Iron Pnictides, Phys. Rev. B 92, 214509 (2015).

[7] Z.-J. Wang, P. Zhang, G. Xu, L.-K. Zeng, H. Miao, X.-Y. Xu, T. Qian, H.-M. Weng, P. Richard, A. V. Fedorov, H. Ding, X. Dai, and Z. Fang, Topological Nature of the $\mathrm{FeSe}_{0.5} \mathrm{Te}_{0.5}$ Superconductor, Phys. Rev. B 92, 115119 (2015).

[8] X.-X. Wu, S.-S. Qin, Y. Liang, H. Fan, and J.-P Hu, Topological Characters in $\mathrm{Fe}\left(\mathrm{Te}_{1-x} \mathrm{Se}_{x}\right)$ Thin Films, Phys. Rev. B 93, 115129 (2016).

[9] G. Xu, B. Lian, P. Tang, X.-L. Qi, and S.-C. Zhang, Topological Superconductivity on the Surface of Fe-Based Superconductors, Phys. Rev. Lett. 117, 047001 (2016).

[10] M. Klug, J. Kang, R. M. Fernandes, and J. Schmalian, Orbital Loop Currents in Iron-Based Superconductors, Phys. Rev. B 97, 155130 (2018).

[11] M. H. Christensen, P. P. Orth, B. M. Andersen, and R. M. Fernandes, Magnetic Degeneracy in Iron Pnictides due to the Interplay between Spin-Orbit Coupling and Quantum Fluctuations, Phys. Rev. Lett. 121, 057001 (2018).

[12] S. V. Borisenko, D. V. Evtushinsky, Z.-H. Liu, I. Morozov, R. Kappenberger, S. Wurmehl, B. Büchner, A. N. Yaresko, T. K. Kim, M. Hoesch, T. Wolf, and N. D. Zhigadlo, Direct Observation of Spin-Orbit Coupling in Iron-Based Superconductors, Nat. Phys. 12, 311 (2016).

[13] M.-W. Ma, P. Bourges, Y. Sidis, Y. Xu, S.-Y. Li, B.-Y. Hu, J.-R. Li, F. Wang, and Y. Li, Prominent Role of Spin-Orbit Coupling in FeSe Revealed by Inelastic Neutron Scattering, Phys. Rev. X 7, 021025 (2017).

[14] D. Hu, W.-L. Zhang, Y. Wei, B. Roessli, M. Skoulatos, L. P. Regnault, G.-F. Chen, Y. Song, H.-Q. Luo, S.-L. Li, and P.-C. Dai, Spin Excitation Anisotropy in the Optimally Isovalent-Doped Superconductor $\mathrm{BaFe}_{2}\left(\mathrm{As}_{0.7} \mathrm{P}_{0.3}\right)_{2}$, Phys. Rev. B 96, 180503(R) (2017).

[15] F. Waßer, C. H. Lee, K. Kihou, P. Steffens, K. Schmalzl, N. Qureshi, and M. Braden, Anisotropic Resonance Modes Emerging in an Antiferromagnetic Superconducting State, Sci. Rep. 7, 10307 (2017).

[16] Y. S. Kushnirenko, A. V. Fedorov, E. Haubold, S. Thirupathaiah, T. Wolf, S. Aswartham, I. Morozov, T. K. Kim, B. Büchner, and S. V. Borisenko, Three-Dimensional Superconducting Gap in FeSe from Angle-Resolved Photoemission Spectroscopy, Phys. Rev. B 97, 180501(R) (2018).

[17] D.-F. Liu et al., Orbital Origin of Extremely Anisotropic Superconducting Gap in Nematic Phase of FeSe Superconductor, Phys. Rev. X 8, 031033 (2018).

[18] R. P. Day, G. Levy, M. Michiardi, B. Zwartsenberg, M. Zonno, F. Ji, E. Razzoli, F. Boschini, S. Chi, R. Liang, P. K. Das, T. Vobornik, J. Fujii, W. N. Hardy, D. A. Bonn, I. E. Elfimov, and A. Damascelli, Influence of Spin-Orbit Coupling in Iron-Based Superconductors, Phys. Rev. Lett. 121, 076401 (2018).

[19] P. Zhang, K. Yaji, T. Hashimoto, Y. Ota, T. Kondo, K. Okazaki, Z.-J. Wang, J.-S. Wen, G. D. Gu, H. Ding, and S. Shin, Observation of Topological Superconductivity on the 
Surface of an Iron-Based Superconductor, Science 360, 182 (2018).

[20] D.-F. Wang et al., Evidence for Majorana Bound States in an Iron-Based Superconductor, Science 362, 333 (2018).

[21] Q. Liu, C. Chen, T. Zhang, R. Peng, Y.-J. Yan, C.-H.-P. Wen, X. Lou, Y.-L. Huang, J.-P. Tian, X.-L. Dong, G.-W. Wang, W.-C. Bao, Q.-H. Wang, Z.-P. Yin, Z.-X. Zhao, and D.-L. Feng, Robust and Clean Majorana Zero Mode in the Vortex Core of High-Temperature Superconductor $\left(\mathrm{Li}_{0.84} \mathrm{Fe}_{0.16}\right)$ OHFeSe, Phys. Rev. X 8, 041056 (2018).

[22] P. Zhang et al., Multiple Topological States in Iron-Based Superconductors, Nat. Phys. 15, 41 (2019).

[23] M. Z. Hasan and C. L. Kane, Topological Insulators, Rev. Mod. Phys. 82, 3045 (2010).

[24] X.-L. Qi and S.-C. Zhang, Topological Insulators and Superconductors, Rev. Mod. Phys. 83, 1057 (2011).

[25] T. Jungwirth, J. Wunderlich, and K. Olejnik, Spin Hall Effect Devices, Nat. Mater. 11, 382 (2012).

[26] D. Pesin and L. Balents, Mott Physics and Band Topology in Materials with Strong Spin-Orbit Interaction, Nat. Phys. 6, 376 (2010).

[27] L. P. Gor'kov and E. I. Rashba, Superconducting 2D System with Lifted Spin Degeneracy: Mixed Singlet-Triplet State, Phys. Rev. Lett. 87, 037004 (2001).

[28] J. M. Lu, O. Zheliuk, I. Leermakers, N. F. Q. Yuan, U. Zeitler, K. T. Law, and J. T. Ye, Evidence for TwoDimensional Ising Superconductivity in Gated $\mathrm{MoS}_{2}$, Science 350, 1353 (2015).

[29] X.-X. Xi, Z.-F. Wang, W.-W. Zhao, J.-H. Park, K. T. Law, H. Berger, L. Forró, J. Shan, and K. F. Mak, Ising Pairing in Superconducting $\mathrm{NbSe}_{2}$ Atomic Layers, Nat. Phys. 12, 139 (2016).

[30] K. Kothapalli, A. E. Bohmer, W. T. Jayasekara, B. G. Ueland, P. Das, A. Sapkota, V. Taufour, Y. Xiao, E. Alp, S. L. Bud'ko, P. C. Canfield, A. Kreyssig, and A. I. Goldman, Strong Cooperative Coupling of PressureInduced Magnetic Order and Nematicity in FeSe, Nat. Commun. 7, 12728 (2016).

[31] J. P. Sun, K. Matsuura, G. Z. Ye, Y. Mizukami, M. Shimozawa, K. Matsubayashi, M. Yamashita, T. Watashige, S. Kasahara, Y. Matsuda, J.-Q. Yan, B. C. Sales, Y. Uwatoko, J.-G. Cheng, and T. Shibauchi, Dome-Shaped Magnetic Order Competing with High-Temperature Superconductivity at High Pressures in FeSe, Nat. Commun. 7, 12146 (2016).

[32] P.-S. Wang, S.-S. Sun, Y. Cui, W.-H. Song, T.-R. Li, R. Yu, H.-C. Lei, and W.-Q. Yu, Pressure Induced Stripe-Order Antiferromagnetism and First-Order Phase Transition in FeSe, Phys. Rev. Lett. 117, 237001 (2016).

[33] S.-H. Baek, D. V. Efremov, J. M. Ok, J. S. Kim, J. V. D. Brink, and B. Büchner, Orbital-Driven Nematicity in FeSe, Nat. Mater. 14, 210 (2015).

[34] A. E. Böhmer, T. Arai, F. Hardy, T. Hattori, T. Iye, T. Wolf, H. V. Löhneysen, K. Ishida, and C. Meingast, Origin of the Tetragonal to Orthorhombic Phase Transition in FeSe: A Combined Thermodynamic and NMR Study of Nematicity, Phys. Rev. Lett. 114, 027001 (2015).

[35] A. V. Chubukov, M. Khodas, and R. M. Fernandes, Magnetism, Superconductivity, and Spontaneous Orbital Order in
Iron-Based Superconductors: Which Comes First and Why?, Phys. Rev. X 6, 041045 (2016).

[36] M.-Q. He, L.-R. Wang, F. Ahn, F. Hardy, T. Wolf, P. Adelmann, J. Schmalian, I. Eremin, and C. Meingast, Dichotomy between In-Plane Magnetic Susceptibility and Resistivity Anisotropies in Extremely Strained $\mathrm{BaFe}_{2} \mathrm{As}_{2}$, Nat. Commun. 8, 504 (2017).

[37] R. M. Fernandes, A. V. Chubukov, J. Knolle, I. Eremin, and J. Schmalian, Preemptive Nematic Order, Pseudogap, and Orbital Order in the Iron Pnictides, Phys. Rev. B 85, 024534 (2012).

[38] R. M. Fernandes, A. V. Chubukov, and J. Schmalian, What Drives Nematic Order in Iron-Based Superconductors?, Nat. Phys. 10, 97 (2014).

[39] See Supplemental Material at http://link.aps.org/ supplemental/10.1103/PhysRevX.10.011034 for details of experimental methods, theoretical analyses, and additional measurement data.

[40] M. Fu, D. A. Torchetti, T. Imai, F. L. Ning, J.-Q. Yan, and A. S. Sefat, NMR Search for the Spin Nematic State in LaFeAsO Single Crystal, Phys. Rev. Lett. 109, 247001 (2012).

[41] R. Zhou, L. Y. Xing, X. C. Wang, C. Q. Jinz, and G.-q. Zheng, Orbital Order and Spin Nematicity in the Tetragonal Phase of the Electron-Doped Iron Pnictides $\mathrm{NaFe}_{1-x} \mathrm{Co}_{x} \mathrm{As}$, Phys. Rev. B 93, 060502(R) (2016).

[42] H. Kontani, T. Saito, and S. Onari, Origin of Orthorhombic Transition, Magnetic Transition, and Shear-Modulus Softening in Iron Pnictide Superconductors: Analysis Based on the Orbital Fluctuations Theory, Phys. Rev. B 84, 024528 (2011).

[43] M. Takigawa, P. C. Hammel, R. H. Heffner, and Z. Fisk, Spin susceptibility in superconducting $\mathrm{YBa}_{2} \mathrm{Cu}_{3} \mathrm{O}_{7}$ from $C u$ Knight Shift, Phys. Rev. B 39, 7371 (1989).

[44] Y. Zhang, M. Yi, Z.-K. Liu, W. Li, J. J. Lee, R. G. Moore, M. Hashimoto, M. Nakajima, H. Eisaki, S.-K. Mo, Z. Hussain, T. P. Devereaux, Z.-X. Shen, and D. H. Lu, Distinctive Orbital Anisotropy Observed in the Nematic State of a FeSe Thin Film, Phys. Rev. B 94, 115153 (2016).

[45] M. D. Watson et al., Emergence of the Nematic Electronic State in FeSe, Phys. Rev. B 91, 155106 (2015).

[46] M. D. Watson, A. A. Haghighirad, H. Takita, W. Mansuer, H. Iwasawa, E. F. Schwier, A. Ino, and M. Hoesch, Shifts and Splittings of the Hole Bands in the Nematic Phase of FeSe, J. Phys. Soc. Jpn. 86, 053703 (2017).

[47] A. I. Coldea and M. D. Watson, The Key Ingredients of the Electronic Structure of FeSe, Annu. Rev. Condens. Matter Phys. 9, 125 (2018).

[48] Z.-P. Yin, K. Haule, and G. Kotliar, Kinetic Frustration and the Nature of the Magnetic and Paramagnetic States in Iron Pnictides and Iron Chalcogenides, Nat. Mater. 10, 932 (2011).

[49] K. Haule and G. Kotliar, Coherence-Incoherence Crossover in the Normal State of Iron Oxypnictides and Importance of Hund's Rule Coupling, New J. Phys. 11, 025021 (2009).

[50] L. Fanfarillo, G. Giovannetti, M. Capone, and E. Bascones, Nematicity at the Hund's Metal Crossover in Iron Superconductors, Phys. Rev. B 95, 144511 (2017). 
[51] P. V. Arribi and L. de'Medici, Hund-Enhanced Electronic Compressibility in FeSe and Its Correlation with $T_{c}$, Phys. Rev. Lett. 121, 197001 (2018).

[52] Y.-Z. You and Z.-Y. Weng, Two-Fluid Description for IronBased Superconductors, New J. Phys. 16, 023001 (2014).

[53] J. Maletz, V. B. Zabolotnyy, D. V. Evtushinsky, S. Thirupathaiah, A. U. B. Wolter, L. Harnagea, A. N. Yaresko, A. N. Vasiliev, D. A. Chareev, A. E. Bohmer, F. Hardy, T. Wolf, C. Meingast, E. D. L. Rienks, B. Büchner, and S. V. Borisenko, Unusual Band Renormalization in the Simplest Iron-Based Superconductor $\mathrm{FeSe}_{1-x}$, Phys. Rev. B 89, 220506(R) (2014).

[54] L. de'Medici, S. R. Hassan, M. Capone, and X. Dai, Orbital-Selective Mott Transition out of Band Degeneracy Lifting, Phys. Rev. Lett. 102, 126401 (2009).

[55] M. Yi et al., Observation of Universal Strong OrbitalDependent Correlation Effects in Iron Chalcogenides, Nat. Commun. 6, 7777 (2015).

[56] M. Yi et al., The Nematic Energy Scale and the Missing Electron Pocket in FeSe, Phys. Rev. X 9, 041049 (2019).

[57] S. Huh et al., Lifted Electron Pocket and Reversed Orbital Occupancy Imbalance in FeSe, arXiv:1903.08360.

[58] M. M. Korshunov, I. Eremin, D. V. Efremov, D. L. Maslov, and A. V. Chubukov, Nonanalytic Spin Susceptibility of a Fermi Liquid: The Case of Fe-Based Pnictides, Phys. Rev. Lett. 102, 236403 (2009).
[59] H. Ghosh, S. Sen, and A. Ghosh, Electronic Origin of Structural Transition in 122 Fe Based Superconductors, J. Phys. Chem. Solids. 102, 157 (2017).

[60] M.-Q. He, L.-R. Wang, F. Ahn, F. Hardy, T. Wolf, P. Adelmann, J. Schmalian, I. Eremin, and C. Meingast, Evidence for Short-Range Magnetic Order in the Nematic Phase of FeSe from Anisotropic In-Plane Magnetostriction and Susceptibility Measurements, Phys. Rev. B 97, 104107 (2018).

[61] F. Yang, S. P. Kou, and Z. Y. Weng, Collective Spin Mode in a Multicomponent System of Coupled Itinerant and Localized Electrons, Phys. Rev. B 81, 245130 (2010).

[62] Y. Z. You, F. Yang, S. P. Kou, and Z. Y. Weng, Magnetic and Superconducting Instabilities in a Hybrid Model of Itinerant/Localized Electrons for Iron Pnictides, Phys. Rev. B 84, 054027 (2011).

[63] Y. Z. You and Z. Y. Weng, Coexisting Itinerant and Localized Electrons in Iron-Based Superconductors, arXiv: 1311.4094.

[64] P. O. Sprau, A. Kostin, A. Kreisel, A. E. Böhmer, V. Taufour, P. C. Canfield, S. Mukherjee, P. J. Hirschfeld, B. M. Andersen, and J. C. S. Davis, Discovery of Orbital-Selective Cooper Pairing in FeSe, Science 357, 75 (2017).

[65] S. Kasahara et al., Field-Induced Superconducting Phase of $\mathrm{FeSe}$ in the BCS-BEC Cross-over, Proc. Natl. Acad. Sci. U.S.A. 111, 16309 (2014). 